Full length article

\title{
Protective therapeutic effects of peptide nanofiber and hyaluronic acid hybrid membrane in in vivo osteoarthritis model
}

\author{
Elif Arslan ${ }^{\mathrm{a}}$, Melis Sardan Ekiz ${ }^{\mathrm{a}}$, Cagla Eren Cimenci ${ }^{\mathrm{a}}$, Nuray Can ${ }^{\mathrm{c}}$, M. Hanifi Gemci ${ }^{\mathrm{c}}$, Huseyin Ozkan ${ }^{\mathrm{c}, *}$, \\ Mustafa O. Guler ${ }^{\mathrm{d}, *}$, Ayse B. Tekinay ${ }^{\mathrm{a}, \mathrm{b}, *}$ \\ a Institute of Materials Science and Nanotechnology, Nanotechnology Research Center (UNAM), Bilkent University, Ankara 06800, Turkey \\ ${ }^{\mathrm{b}}$ Neuroscience Graduate Program, Bilkent University, Ankara 06800, Turkey \\ ${ }^{\mathrm{c}}$ Department of Orthopedics and Traumatology, Gulhane Military Medical Academy, Ankara 06010, Turkey \\ ${ }^{\mathrm{d}}$ Institute for Molecular Engineering, University of Chicago, Chicago, IL 60637, USA
}

\section{A R T I C L E I N F O}

\section{Article history:}

Received 7 October 2017

Received in revised form 2 April 2018

Accepted 5 April 2018

Available online 12 April 2018

\section{Keywords:}

Osteoarthritis model

Peptide gel

Hyaluronic acid

Hybrid membrane

Protection

\begin{abstract}
A B S T R A C T
Osteoarthritis $(\mathrm{OA})$ is a condition where tissue function is lost through a combination of secondary inflammation and deterioration in articular cartilage. One of the most common causes of OA is agerelated tissue impairment because of wear and tear due to mechanical erosion. Hyaluronic acid-based viscoelastic supplements have been widely used for the treatment of knee injuries. However, the current formulations of hyaluronic acid are unable to provide efficient healing and recovery. Here, a nanofiberhyaluronic acid membrane system that was prepared by using a quarter of the concentration of commercially available hyaluronic acid supplement, Hyalgan ${ }^{\circledR}$, was used for the treatment of an osteoarthritis model, and Synvisc ${ }^{\circledR}$, which is another commercially available hyaluronic acid containing viscoelastic supplement, was used as a control. The results show that this system provides efficient protection of arthritic cartilage tissue through the preservation of cartilage morphology with reduced osteophyte formation, protection of the subchondral region from deterioration, and maintenance of cartilage specific matrix proteins in vivo. In addition, the hybrid nanofiber membrane enabled chondrocyte encapsulation and provided a suitable culturing environment for stem cell growth in vitro. Overall, our results suggest that this hybrid nanofibrous scaffold provides a potential platform the treatment of OA.
\end{abstract}

\section{Statement of Significance}

Osteoarthritis is a debilitating joint disease affecting millions of people worldwide. It occurs especially in knees due to aging, sport injuries or obesity. Although hyaluronic acid-based viscoelastic supplements are widely used, there is still no effective treatment method for osteoarthritis, which necessitates surgical operation as an only choice for severe cases. Therefore, there is an urgent need for efficient therapeutics. In this study, a nanofiber-HA membrane system was developed for the efficient protection of arthritic cartilage tissue from degeneration. This hybrid nanofiber system provided superior therapeutic activity at a relatively lower concentration of hyaluronic acid than Hyalgan ${ }^{\circledR}$ and Synvisc ${ }^{\circledR}$ gels, which are currently used in clinics. This work demonstrates for the first time that this hybrid nanofiber membrane scaffold can be utilized as a potential candidate for osteoarthritis treatment.

(C) 2018 Acta Materialia Inc. Published by Elsevier Ltd. All rights reserved.
* Corresponding authors at: Department of Orthopedics and Traumatology, Gulhane Military Medical Academy, Ankara 06010, Turkey (H. Ozkan). Institute for Molecular Engineering, University of Chicago, Chicago, IL 60637, USA (M.O. Guler). Neuroscience Graduate Program, Bilkent University, Ankara 06800, Turkey (A.B. Tekinay).

E-mail addresses: heorto@yahoo.com (H. Ozkan), mguler@uchicago.edu (M.O. Guler), atekinay@bilkent.edu.tr (A.B. Tekinay).

\section{Introduction}

Cartilage tissue is distributed widely in the human body and plays essential roles in joint lubrication and the transmission of loads [1]. Chondrocytes are cartilage-specific cells that oversee the turnover of the non-cellular components of this tissue, but cannot repair major cartilage defects due to their low capacity for renewal [1]. Consequently, chondrocytes are assisted by mesenchymal stem cells (MSCs) that migrate to the defect site from 
the subchondral region during cartilage regeneration. This complex and lengthy repair process, in conjunction with repeated exposure to mechanical loading, make cartilage defects highly susceptible to arthritic degeneration, which is one of the most common diseases of cartilage tissue. Osteoarthritis (OA) is a condition in which tissue function is lost through a combination of secondary inflammation, deterioration and erosion in joint cartilage [2]. Genetic, metabolic and biochemical factors all contribute to the etiology of OA, however, one of the most common causes of $\mathrm{OA}$ is age-related tissue impairment. On the other hand, joint problems may also occur in earlier ages due to causes such as traumatic accidents or obesity $[3,4]$. Radiological analyses have demonstrated that OA typically develops within less than 10 years in patients with untreated early cartilage injuries [5].

Approximately $70 \%$ of cartilage tissue is composed of water by volume [6]. Proteoglycans interact with water, water-soluble molecules and various growth factors through their glycosaminoglycan (GAG) moieties, such as the negatively-charged heparan sulfate, chondroitin sulfate, and hyaluronic acid [7]. GAGs electrostatically interact with growth factors to stabilize them and increase their availability to cells in aqueous media [7]. Collagen II is the most common fibrillar protein in cartilage and forms the functional skeleton of this tissue with collagen IX and collagen XI, which provides mechanical support and imparts cartilage with the physical features required for its proper functioning [8-10]. Motifs on matrix proteins such as collagen and fibronectin, in conjunction with growth factors associated with GAGs, activate various intracellular signaling pathways through their interactions with cellular receptors, thereby initiating specific cellular responses [11-13]. During OA formation, collagen degradation increases, proteoglycan content decreases, the prevalence of individual proteoglycans is altered, and water content increases $[14,15]$. In addition, the amount and molecular size of hyaluronic acid in the synovial fluid is also reduced. Since OA alters the whole structure and function of cartilage tissue in a highly irreversible manner, the early prevention of this disease is critical for increasing the life quality of prospective patients. However, none of the current treatment methods used can effectively provide healthy cartilage tissue formation [16-18].

Bone marrow stimulation techniques such as arthroscopic debridement, lavage and microfracture; osteochondral autograft and allograft techniques; and autologous chondrocyte implantation (ACI) and matrix-assisted ACI (MACI) are the methods that are used to repair cartilage [19-21]. Today, these methods are used to ameliorate the symptoms of cartilage damage and partially restore mobility [22]. However, due to the mechanical weakness of the newly formed tissue, degeneration persists and eventually propagates into healthy cartilage even with treatment [23]. In addition, difficult surgical operations, and high-cost and ineffectiveness of the therapeutics used in clinics necessitate new therapeutic strategies. Therefore, tissue engineering applications employing three-dimensional scaffold-matrix systems that allow cellular infiltration and in situ differentiation such as MACI applications are promising for the development of new approaches to provide long-term joint function and durability [24,25]. For this purpose, sustaining molecular components of the cartilage tissue and its structure with proper biochemical signals and mechanical support is critical for the regeneration of damaged tissue.

In this study, we developed a hybrid peptide nanofiber-HA membrane and examined its therapeutic effect in an in vivo OA model in rats. At the liquid-liquid interface, the negatively charged high molecular weight HA solution contacts with positively charged peptide amphiphile (PA) molecules, which leads to the formation of a solid membrane. When two solutions are brought into contact, a physical layer is formed through the initial electrostatic complexation of the molecules, which provides the formation of hierarchically organized hybrid nanofibers. Hence, during the maturation of membrane, a unique scaffold structure develops, which contains three regions: an amorphous biopolymer layer, a layer of PA nanofibers parallel to the interface, and a layer of hybrid fibers perpendicular to the interface [26,27]. Through this process, the nanofibers form micro-scale three-dimensional networks that are mechanically stable in aqueous media. Here, we established hybrid nanofiber membranes by utilizing K-PA and Hyalgan ${ }^{\circledR}$, which provided both the mechanical strength to support tissue and the bioactive signals for the activation of chondrocyte proliferation and functional collagen II protein synthesis. After the in vitro evaluation of hybrid nanofiber membranes using stem cell viability and chondrocyte proliferation assays under 2D and 3D environments, the protective capacity of these membranes were investigated in vivo using early-stage and late-stage rat OA models. HA/K-PA injection was performed intra-articularly to fill joint cavity, and interacted with cartilage surface on the condyles. The therapeutic effect of this hybrid nanofiber membrane was found to be superior to the commercially available Hyalgan ${ }^{\circledR}$ and Synvisc ${ }^{\circledR}$ gel at a lower concentration and provides a facile and feasible alternative to existing treatment methods for OA.

\section{Experimental section}

\subsection{Materials}

9-Fluorenylmethoxycarbonyl (Fmoc) and tertbutoxycarbonyl (Boc) protected amino acids, [4-[ $\alpha$ - $\left(2^{\prime}, 4^{\prime}\right.$-dimethoxyphenyl) Fmoc aminomethyl]enoxy] acetamidonorleucyl-MBHA resin (Rink amide MBHA resin), Fmoc-Glu(OtBu)-Wang resin and 2-(1H-benzotria zol-1-yl)-1,1,3,3-tetramethyluronium hexafluorophosphate (HBTU) were purchased from NovaBiochem and ABCR. All other chemicals and materials used were analytical grade and were obtained from Invitrogen, Fisher, Merck, Alfa Aesar and SigmaAldrich. Cover glasses and tissue culture plates (24-well) were purchased from Deckglaser and BD. Hyalgan ${ }^{\circledR}$ and Synvisc (Hylan G_F20) ${ }^{\circledR}$ (cross-linked version of high molecular weight hyaluronic acid) were commercially purchased. Chondrogenic differentiation media was purchased from Sigma. Live/Dead Assay (L3224), Alamar Blue and other cell culture materials were purchased from Invitrogen. Antibodies were purchased from Abcam and Millipore.

\subsection{Synthesis and characterization of peptide amphiphile molecules}

The peptide amphiphile molecule, Lauryl-Val-Val-Ala-Gly-LysAm (K-PA), was synthesized by Fmoc solid phase peptide synthesis method. Rink amide MBHA resin was used as solid support for $\mathrm{K}$ PA. For the amino acid coupling reactions, 2 equivalents of Fmocprotected amino acid, 1.95 equivalents of HBTU and 3 equivalents of $\mathrm{N}, \mathrm{N}$-diisopropylethylamine (DIEA) mixed in dimethylformamide (DMF) for $3 \mathrm{~h}$. 20\% piperidine/DMF solution was used for Fmoc deprotection, while 10\% acetic anhydride-DMF solution was used for $20 \mathrm{~min}$ for the acetylation of the unreacted amine groups after each coupling step. The terminal lauryl group was coupled by mixing lauric acid in a manner analogous to amino acid attachment. After couplings were finished, the cleavage of the peptides from the resin and deprotection of acid-labile protected amino acids were performed by a mixture of trifluoroacetic acid (TFA):triisopropylsilane (TIS):water in a ratio of 95:2.5:2.5 for $2.5 \mathrm{~h}$. Then, rotary evaporation was performed to remove excess TFA. Ice-cold diethyl ether was used to triturate the remaining residue and the resulting white pellet was freeze-dried. 


\subsubsection{Liquid chromatography and mass spectrometry (LC-MS)}

Peptide amphiphile molecules were identified and analyzed by reverse phase HPLC on an Agilent 6530 accurate-Mass Q-TOF LC/ MS equipped with an Agilent 1200 HPLC. K-PA was purified on an Agilent 1200 HPLC system by using a Zorbax prepHT 300CBC8 column with a water-acetonitrile $(0.1 \%$ TFA) gradient.

\subsubsection{Preparation and characterization of self-assembled peptide amphiphile-HA hybrid nanofiber membrane}

For nanofiber formation, K-PA was mixed with Hyalgan ${ }^{\circledR}$ at equal millimolar $(5 \mathrm{mM})$ ratio in order to form a HA/K-PA hybrid membrane. The approximate molecular weight for Hyalgan ${ }^{\circledR}$ was determined as $615,000 \mathrm{Da}$ according to the manufacturer's guidelines.

\subsubsection{Transmission electron microscopy imaging}

TEM analysis of HA/K-PA hybrid membrane was performed with a FEI Tecnai G2 F30 TEM at $200 \mathrm{kV}$. For STEM mode images, a high-angle annular dark field (HAADF) detector was used. 5 mM HA/K-PA nanofiber system was diluted 100-200 times and then dropped on a 200-mesh copper TEM grid. Samples were left at room temperature for $7 \mathrm{~min}$, stained by $2 \mathrm{wt} \%$ uranyl acetate for another $2 \mathrm{~min}$ and air dried prior to TEM imaging.

\subsubsection{Circular dichroism (CD) analyses}

CD measurements were performed by Jasco J-815 CD spectrophotometer for the analysis of the secondary structure of the HA/K-PA membrane and Hyalgan ${ }^{\circledR}$ gel. All samples were measured at 100 -fold dilutions of concentrations used in in vitro experiments. For each measurement, $300 \mu \mathrm{L}$ of PA material was transferred into a $1 \mathrm{~mm}$ quartz cuvette, which was inverted gently to mix the sample without damaging the assembled nanostructures. Spectra were obtained at room temperature at a range of $300-190 \mathrm{~nm}$, with a data interval of $1 \mathrm{~nm}$ and a scanning speed of $100 \mathrm{~nm} / \mathrm{min}$. The results were expressed as mean residue ellipticity and converted to the unit of deg. $\mathrm{cm}^{2} \cdot \mathrm{dmol}^{-1}$.

\subsubsection{Oscillatory rheology analysis}

Oscillatory rheology measurements were performed with an Anton Paar Physica MCR301 system for the analysis of the mechanical characteristics of the HA/K-PA hybrid membrane. A $25 \mathrm{~mm}$ parallel plate with a gap distance of $0.5 \mathrm{~mm}$ was used at $25^{\circ} \mathrm{C}$ for all measurements. The total membrane volume was adjusted as $250 \mu \mathrm{L}$. Hybrid membrane was freshly prepared as $1: 1$ ratio mixture of $5 \mathrm{mM}$ K-PA and $5 \mathrm{mM}$ Hyalgan ${ }^{\circledR}$, and the mixture was then sonicated for $30 \mathrm{~min}$. The upper plate was adjusted to the $0.5 \mathrm{~mm}$ position and the membrane was incubated in this position for 15 min prior to measurement. For strain sweep measurements, angular frequency was kept constant at $10 \mathrm{rad} / \mathrm{s}$, and strain was increased between 0.1 and $100 \%$. Storage and loss moduli were recorded at each strain value. All rheology measurements were performed on membrane with and without cells with three replicates.

\subsubsection{SEM analysis}

For SEM imaging, $1 \%(\mathrm{w} / \mathrm{v})$ bulk peptide nanofiber membranes were prepared on silicon wafers. Samples were dehydrated in graded ethanol solutions, starting with $20 \%$ ethanol and proceeding to absolute ethanol for $10 \mathrm{~min}$ at each step. Samples were dried with a Tousimis Autosamdri-815B critical point drier, coated with $10 \mathrm{~nm} \mathrm{Au} / \mathrm{Pd}$ and imaged with a FEI Quanta 200 FEG SEM.

\subsection{In vitro cell culture experiments}

The rMSCs (rat mesenchymal stem cells, Invitrogen S1601-100) at passage 3 (post-thaw) were used in 2D cell culture experiments.
These cells maintain their stem cell characteristics until passage number 5 (post-thaw). The rMSCs were initially cultured in the manufacturer's recommended cell media which contains DMEM with GlutaMAX (Gibco) and supplemented with $10 \%$ fetal bovine serum (FBS-Gibco), in tissue culture plates at standard culture conditions (at $37{ }^{\circ} \mathrm{C}$ under $5 \% \mathrm{CO}_{2}$ ). Growth factors were not included into cell media.

Rat chondrocytes were isolated according to a protocol adapted from Gosset et al. [28]. All procedures on animals were approved by the Gulhane Military Medical Academy (GATA) Animal Ethics Committee. Briefly, femoral heads, femoral condyles and tibial plateau were isolated from one rat and placed in 1X PBS. Then, pieces of cartilage were incubated in $10 \mathrm{~mL}$ digestion solution for $45 \mathrm{~min}$ at $37^{\circ} \mathrm{C}$ in a thermal incubator. After retrieving cartilage pieces using a $25 \mathrm{~mL}$ pipette, tissue fragments were agitated using a 25 $\mathrm{mL}$ pipette to detach soft tissues in $0.5 \mathrm{mg} \mathrm{mL}^{-1}$ collagenase $\mathrm{D}$ solution. Then, cartilage pieces were retrieved and placed in 10 $\mathrm{mL}$ of $0.5 \mathrm{mg} \mathrm{ml}^{-1}$ collagenase D solution for overnight at $37^{\circ} \mathrm{C}$. On the next day, the collagenase $\mathrm{D}$ solution containing residual cartilage was retrieved, and the cell suspension was passed through a cell strainer (Falcon ${ }^{\mathrm{TM}}$ ) and centrifuged for $10 \mathrm{~min}$ at $400 \mathrm{~g}$ at $20^{\circ} \mathrm{C}$. Then, cells were seeded into culture medium at a density of $8 \times 10^{3}$ cell per $\mathrm{cm}^{2}$. The cell media was prepared as recommended by Gosset [28], which contains DMEM with $2 \mathrm{mM} \mathrm{L-}$ Gln (Invitrogen), $50 \mathrm{U} \mathrm{ml}^{-1}$ penicillin and $0.05 \mathrm{mg} \mathrm{ml}^{-1}$ streptomycin, supplemented with 10\% FBS (Gibco).

For in vitro studies, both 2D and 3D cell culture methods were performed to investigate the effect of bioactive hybrid membranes on both stem cells and chondrocytes. For 2D in vitro cell culture experiments, HA/K-PA ( $1 \mathrm{mM}$ : $1 \mathrm{mM})$ membranes were prepared in wells and dried overnight. On the next day, rMSCs (passage 8) were seeded on the coatings. Uncoated tissue culture plate (TCP) was used as control group. For 3D in vitro cell culture experiments, rat chondrocyte cells were seeded in 3D peptide hybrid nanofiber membrane scaffolds prepared by the mixture of $5 \mathrm{mM}$ Hyalgan ${ }^{\circledR}$ and $5 \mathrm{mM}$ K-PA at a 1:1 ratio. Each solution was prepared in $\mathrm{ddH}_{2}-$ O. During 3D encapsulation of chondrocytes in HA/K-PA gel, primary chondrocytes were initially grown in a flask as 2D. Then, the cells were trypsinized and collected. For each scaffold preparation, the concentrations of the cell suspensions were determined, and cells were seeded by mixing this cell suspension with corresponding Hyalgan ${ }^{\circledR}$ or Synvisc ${ }^{\circledR}$. For HA/K-PA gel, this Hyalgan ${ }^{\circledR}$ and cell mixture was mixed with K-PA solution, which resulted in 3D cell encapsulated hydrogel. For Hyalgan ${ }^{\circledR}$ and Synvisc ${ }^{\circledR}$ (Hylan G_F20) controls, chondrocyte cell suspensions were mixed directly in Hyalgan ${ }^{\circledR}$ or Synvisc ${ }^{\circledR}$. Following scaffold preparation, $3 \mathrm{~mL}$ of cell medium was added to the wells and the scaffolds were incubated for $30 \mathrm{~min}$ at $37^{\circ} \mathrm{C}$.

Cell viability was analyzed by Alamar Blue (Invitrogen) and Live/Dead (Life Technologies) assays. $5 \times 10^{5}$ rMSCs were seeded onto nanofiber membranes and their viability was measured at days 1,2 , and 3 by quantifying the fluorescence and absorbance of the reagent using spectrophotometry for the Alamar Blue assay, and imaging the cells by fluorescence microscopy and confocal microscopy for the Live/Dead Assay. In addition, for the biocompatibility analyses of the 3D hybrid membrane scaffold, chondrocytes were encapsulated into hybrid nanofiber membranes and Live/ Dead assay was performed.

\subsection{In vivo osteoarthritis model in rat and intra-articular hybrid nanofiber membrane treatment}

All procedures on animals were approved by the Gulhane Military Medical Academy (GATA) Animal Ethics Committee. ARRIVE guideline for the in vivo experimental part has been included as supplementary information. All animals were allowed free access 
Table 1

In vivo experimental plan.

\begin{tabular}{|c|c|c|c|c|}
\hline Experimental Groups & OA Model Surgery & $\begin{array}{l}\text { Intra-articular Injected Experimental } \\
\text { Groups }\end{array}$ & $\begin{array}{l}\text { Animal } \\
\text { Amount }\end{array}$ & Tissue Extractions \\
\hline $\begin{array}{l}\text { Early OA Groups } \\
1 \text { week/6 week } \\
(1 \mathrm{~W} / 6 \mathrm{~W})\end{array}$ & $\begin{array}{l}\text { Medial Meniscus Destabilization } \\
\text { Model } \\
1 \text { week waiting time after surgery }\end{array}$ & $\begin{array}{l}\text { HA/K-PA } \\
\text { Synvisc }^{\circledR} \\
\text { Hyalgan }^{\circledR} \\
\text { Sham (Physiological Saline) }\end{array}$ & $\begin{array}{l}6 \\
6 \\
6 \\
6\end{array}$ & $\begin{array}{l}\text { Intra-articular Injection of scaffolds and } \\
\text { saline } \\
6 \text { weeks waiting time after injections }\end{array}$ \\
\hline $\begin{array}{l}\text { Late OA Groups } \\
6 \text { week/6week (6W/ } \\
6 \mathrm{~W})\end{array}$ & $\begin{array}{l}\text { Medial Meniscus Destabilization } \\
\text { Model } \\
6 \text { weeks waiting time after surgery }\end{array}$ & $\begin{array}{l}\text { HA/K-PA } \\
\text { Synvisc }^{\circledR} \\
\text { Hyalgan }^{\circledR} \\
\text { Sham (Physiological Saline) }\end{array}$ & $\begin{array}{l}6 \\
6 \\
6 \\
6\end{array}$ & $\begin{array}{l}\text { Intra-articular Injection of scaffolds and } \\
\text { saline } \\
6 \text { weeks waiting time after injections }\end{array}$ \\
\hline Healthy Control & $\begin{array}{l}\text { Healthy right knees (un-operated) of } \\
\text { animals }\end{array}$ & No injection & 6 & $\begin{array}{l}\text { Healthy tissues extracted at same time with } \\
\text { other leg }\end{array}$ \\
\hline
\end{tabular}

of water and food, and they were housed in optimum conditions according to animal facility (GATA Animal Facility) regulations. 48 Sprague-Dawley (Rattus norvegicus, GATA Animal Laboratory, Ankara, Turkey) male rats (weight 400-450 g) were divided into 8 groups of 6 rats each as 4 groups for early stage OA model and 4 groups for late stage OA model (Table 1 ). Sample size calculations were done by biostatistics expert who was ethical committee member. The minimum number of animals were calculated as 48 to achieve power $=0.8$ and alpha $=0.05$ by using Gpower V3.13 program. All rats were randomly allocated to groups. For the development of OA in rats, medial meniscus destabilization was performed by the excision of connective tissues between femur and tibial bones in the knees of rats and osteoarthritis formation was observed due to cartilage wear. 6 rats were used for each group. Each rat was anesthetized by general anesthesia with ketaminexylazine via intramuscular injection. Rats' knees were incised longitudinally from the upper patella to the tuberositas tibia, followed by arthrotomy with the medial parapatellar. Then, locations of the anterior medial meniscus and anterior meniscotibial ligaments were determined, and the anterior medial meniscotibial ligament was excised to destabilize the medial meniscus. The joint incisions were closed after observing that the medial meniscus had lost its stability and moved after the excision. For early OA model, sample injections were performed 1 week after the surgery, whereas for late OA groups, injections were performed 6 weeks after surgery. A total volume of $300 \mu \mathrm{L}$ was injected with corresponding scaffolds by insulin needle through intra-articular injection. Experimental groups are HA/K-PA, Synvisc ${ }^{\circledR}$, Hyalgan ${ }^{\circledR}$, Sham (physiological saline injected group). All animals were sacrificed 6 weeks after injection, and tissue was examined by the histopathological analysis of cartilage tissues. Healthy right knees of operated animals were used as healthy tissue control.

\subsection{Histological and immunohistochemical stainings of tissue sections}

Samples were fixed in $4 \%$ paraformaldehyde for $48 \mathrm{~h}$ at $4{ }^{\circ} \mathrm{C}$ and decalcified in $5 \%$ formic acid. $5 \%$ formic acid solution was changed every 2-3 days. The completion of decalcification was periodically tested with ammonium oxalate test. Decalcified samples were dehydrated in a graded series of ethanol and cleared in two changes of xylene. Samples were embedded in paraffin blocks and sectioned at 7-10 $\mu \mathrm{m}$ thickness by microtome. For histological and immunohistochemical evaluations, sections were deparaffinized and rehydrated through a series of graded alcohol solutions. Defect was monitored by the staining of representative slides taken at 20 section $(\sim 200 \mu \mathrm{m})$ intervals to determine the extent of cartilage degeneration through anterior to posterior regions of cartilage. For GAG imaging, sections were stained with Safranin$\mathrm{O}$ for $5 \mathrm{~min}$ and Fast Green for $5 \mathrm{~min}$. Masson's trichrome staining was performed to visualize the mature bone collagens, muscles, and collagens found in cartilage. For staining, after deparaffiniza- tion, slides were stained with hematoxylin for $45 \mathrm{~s}$, Biebrich Scarlett for $5 \mathrm{~min}$, phosphomolibdic acid/phosphotungistic acid solution for $5 \mathrm{~min}$, and aniline blue for $5 \mathrm{~min}$. Sirius red (Direct Red) staining was performed to visualize the organization of collagen fibers in the tissues. For staining, after deparaffinization, slides were stained with Weigert's hematoxylin for $8 \mathrm{~min}$ and pico-sirius red for $1 \mathrm{~h}$. Then sections dehydrated in graded ethanol solutions and cleared in xylene. Slides were mounted by Histomount ${ }^{\circledR}$ mounting medium and imaged by light microscopy (Zeiss, Axio Scope).

For immunohistochemical stainings, slides were treated with Pepsin (Sigma) enzyme for antigen retrieval to uncover epitopes for $15 \mathrm{~min}$ at $37^{\circ} \mathrm{C}$ after rehydration steps, then hydrogen peroxide was used to prevent endogenous peroxidase activity at room temperature for $20 \mathrm{~min}$. After blocking for $2 \mathrm{~h}$ at room temperature, sections were incubated with primary Collagen II antibody (Abcam-ab185430) at $4{ }^{\circ} \mathrm{C}$ overnight. Sections were then washed extensively with TBS $\mathrm{w} /$ Triton-X $(0.01 \% \mathrm{vol} / \mathrm{vol})$ and treated with Goat anti-Mouse IgG-HRP (Millipore-12-349) at a dilution of 1:500 for $1 \mathrm{~h}$ at room temperature to detect bound primary antibodies. Secondary antibody binding was visualized with diaminobenzidine and nuclei were stained with hematoxylin (Sigma-Aldrich). Collagen II antibody was tested on positive control samples before staining of sections from experimental groups. Negative controls were obtained by omitting primary antibody and incubating with $1 \%$ normal goat serum/TBS. Slides were mounted with Histomount ${ }^{\circledR}$ mounting medium.

\subsubsection{Histological scoring}

The histopathological assessment of OA was performed to determine the extent of tissue protection from degeneration following hybrid nanofiber membrane treatment. A validated Osteoarthritis Research Society International (OARSI) scoring system was employed for the evaluation of the therapeutic potential of hybrid nanofiber membranes (Table S1, supporting file) [29]. The images were blinded, and were scored by trained researchers. Tissue sections from 6 rats per experimental group were scored from anterior to posterior at 20 sections interval $(\sim 200 \mu \mathrm{m})$. The medial regions of cartilage tissue and subchondral bone were analyzed by histopathological evaluation. As an uninjured control, the healthy tissues extracted from healthy legs (un-operated) of rats were analyzed. OARSI scoring method includes the evaluation of cartilage matrix loss width, cartilage degeneration score, total cartilage degeneration width, significant cartilage degeneration width, zonal depth ratio of lesions, osteophyte development and calcified cartilage and subchondral bone damage score in four major categories; the nature of the predominant tissue, structural characteristics, cellular evidence of degeneration and degenerative changes in adjacent cartilage. Cartilage degeneration was graded as: No degeneration: 0 ; Minimal degeneration (5-10\% of the total projected cartilage area affected by matrix or chondrocyte loss): 
1; Mild degeneration (11-25\% affected): 2; Moderate degeneration (26-50\% affected): 3; Marked degeneration (51-75\% affected): 4; Severe degeneration (greater than 75\% affected): 5 .

\subsection{Statistical analysis}

All data are presented as mean \pm SEM (standard error of mean). The significance of differences between groups was determined with Student's $t$-test (two-tailed) and one-way analyses of variance (ANOVA) with Bonferroni posttest. Differences were considered significant at $\mathrm{p}<0.05$. For immunohistochemical scoring analyses, statistical units are scores of tissue slides from anterior-toposterior region of cartilage tissue of each animal for all experimental groups. All in vitro experiments were performed with at least five replicas, and in vivo experiments were performed with at least five on replicas.

\section{Results}

\subsection{Synthesis and characterization of hybrid nanofiber membranes}

In this study, hybrid peptide nanofiber - hyaluronic acid membranes were formed to create an effective scaffold system for the protection from degeneration of osteoarthritic cartilage tissue. The synergistic effect of the nanofibrous morphology of peptide amphiphiles and bioactivity of hyaluronic acid molecules were combined to create a mechanically stable nanofiber microenvironment. In addition, commercially available Hyalgan ${ }^{\circledR}$ gel and Synvisc $^{\circledR}$ gel (the cross-linked counterpart of hyaluronic acid) were used as positive controls for in vivo studies. For the formation of hybrid peptide amphiphile-hyaluronic acid membrane scaffold,
K-PA and Hyalgan ${ }^{\circledR}$ solutions were used. All PA molecules were produced by solid-phase peptide synthesis, and they were characterized and purified by LC-MS and preparative HPLC prior to use (Fig. S1). According to LC-MS results, the purity of PA molecules was more than $95 \%(\mathrm{w} / \mathrm{w})$ after HPLC. STEM (scanning transmission electron microscopy) of peptide nanofibers (Fig. 1B) revealed that hybrid nanofiber networks were formed through the coassembly of K-PA and Hyalgan ${ }^{\circledR}$ into nanofibers with high-aspect ratios and nanometer-scale diameters (10-20 nm) through electrostatic interactions between positively and negatively charged building blocks presented in each molecule. Indeed, the sizes of the hybrid nanofibers were similar to the fibrous collagen and glycoproteins found in the cartilage ECM [30,31]. In addition, SEM (scanning electron microscopy) analyses of membranes (Fig. 1C) confirmed the formation of porous nanofiber networks within the membrane structure. Chemical structures of the PA molecules and hyaluronic acid are shown in Fig. 1A. The secondary structures of the networks were characterized by $\mathrm{CD}$ spectroscopy. According to CD spectra, hybrid nanofiber membranes (HA/K-PA) had $\beta$-sheet conformation with bands at $\sim 220 \mathrm{~nm}$ (Fig. 1E). On the other hand, pure K-PA and Hyalgan ${ }^{\circledR}$ molecules were not observed to form $\beta$ sheets at the analyzed concentrations. Oscillatory rheology analyses were also performed to investigate the mechanical properties of hybrid nanofiber membranes. The hybrid membrane was formed at nanoscale to microscale, which resulted in hierarchically organized hybrid nanofibers that formed a hydrolytically stable gel in aqueous environment (Fig. 1D). In line with the previous studies, rheological properties of this structure were investigated through time sweep and strain sweep tests to evaluate the mechanical properties of hybrid nanofibers [27]. Time sweep and strain sweep oscillatory rheology analyses were conducted in order to assess the
A

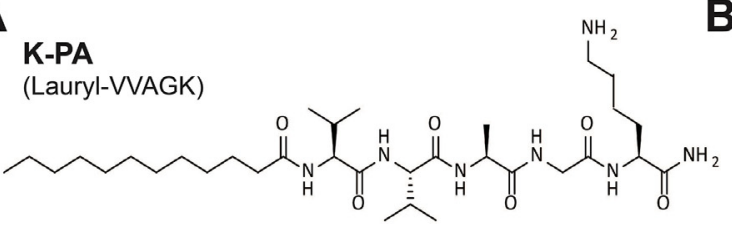

Hyaluronic Acid

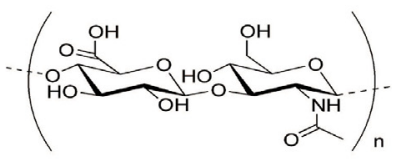

D

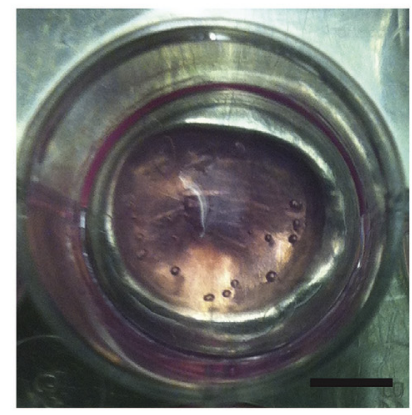

B

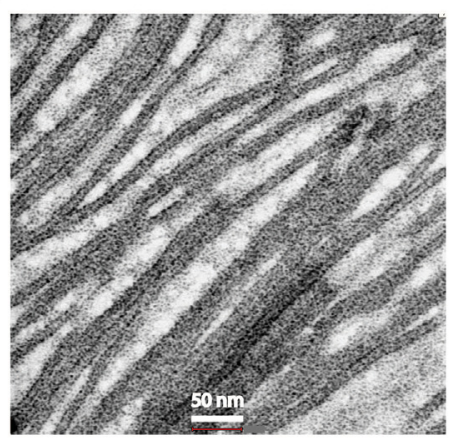

E

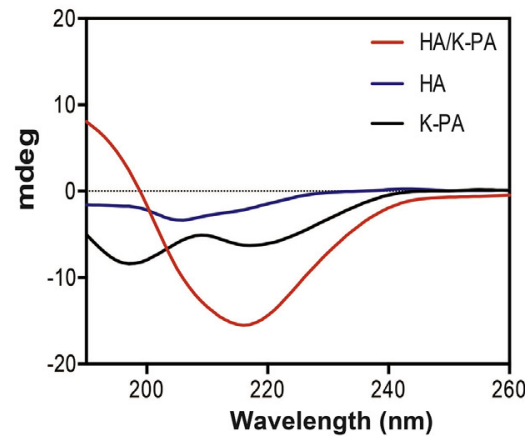

C

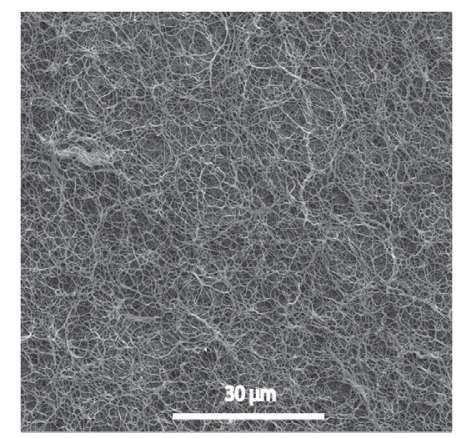

$\mathbf{F}$

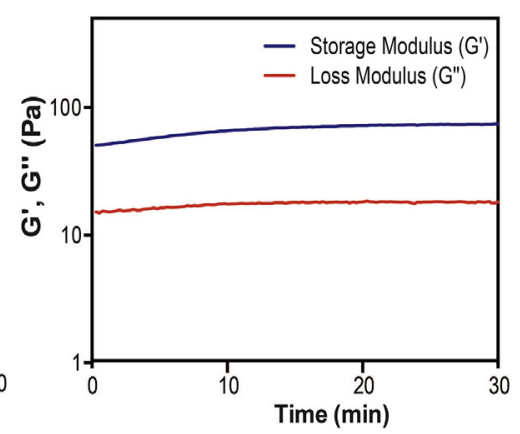

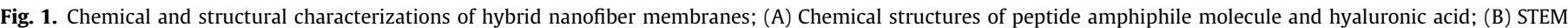

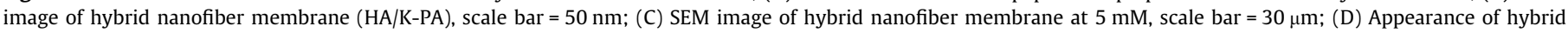

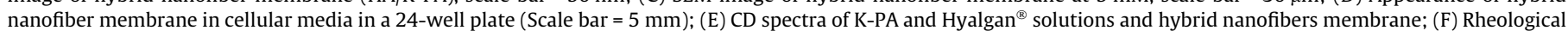
analysis of hybrid nanofiber membrane. 
gel formation mechanics and viscoelastic properties of the scaffold material (Fig. 1F and Fig. S2). Hybrid nanofibrous structure demonstrated viscoelastic properties as time sweep reflects the growth of gel's strength and structure during gel formation. The storage modulus of the hybrid nanofiber membrane was found to be higher than its loss modulus at in vivo experimental concentrations, suggesting that the material was hydrolytically stable and exhibited robust mechanical features.

\subsection{Hybrid nanofiber membrane provide a biocompatible microenvironment for MSC and chondrocyte culturing and encapsulation in $2 D$ and $3 D$ in vitro experiments}

In vitro investigation of membranes is vital for assessing their biocompatibility and capability to be used for the encapsulation of cells. Hence, biocompatibility of nanofiber membranes was evaluated through both 2D and 3D cell culturing. 2D in vitro cell culturing of mesenchymal stem cells (MSC) revealed that hybrid nanofiber membranes presented a suitable environment for the attachment and growth of stem cells (Fig. 2A \& B).

In addition, 3D in vitro chondrocyte culturing was performed to evaluate the potential use of membranes for improved ACI (autologous chondrocyte implantation) therapy (Fig. 2C). According to Live/Dead assay results, the hybrid nanofiber (HA/K-PA) membrane was biocompatible and supported the growth and proliferation of chondrocytes in 3D microenvironments (Fig. 2C). However, neither Hyalgan ${ }^{\circledR}$ nor Synvisc ${ }^{\circledR}$ scaffolds were mechanically stable as they dissolved in cell media and did not provide cell encapsulation for 3D chondrocyte culturing; rather, we observed 2D cellular adhe- sion similar to TCP (tissue culture plate) control in these wells. Hybrid nanofibrous scaffold was compared with Hyalgan ${ }^{\circledR}$ and Synvisc ${ }^{\circledR}$ in vitro as like in vivo since they have biological signals presented to mediate cellular responses. There are other types of HA gels that used for cell delivery as well, but they are mostly chemically modified research products which were unsuitable to compare with hybrid system [32]. Therefore, HA/K-PA hybrid nanofibrous gel was compared with these scaffolds that this hybrid system was originated (HA/K-PA is mixture of Hyalgan ${ }^{\circledR}$ and K-PA).

When considering the importance of 3D microenvironments for cellular behaviors, it is essential to design and recruit mechanically stable scaffolds for further clinical applications. Our 3D hybrid nanofiber scaffold presents a highly porous structure that provides larger surface areas for cell attachment and proliferation, which enables the use of higher amounts of cells in clinical applications [33]. In addition, this hybrid nanofiber system is much closer to the native tissue morphology than $2 \mathrm{D}$ cell culture.

\subsection{Hybrid nanofiber membrane promotes the protection of cartilage tissue in osteoarthritic rat knees}

For the investigation of cartilage tissue, an optimization study was performed before the main experiment to choose the proper surgical model for OA formation, proper time period, and working gel concentration (Fig. S6). Three different surgical models, medial meniscus destabilization, anterior cruciate ligament dissection, and meniscus excision, were evaluated in rats. In line with the previous studies, medial meniscus destabilization model was found to be the optimum model resulting in mild osteoarthritis at medial

\section{D In vitro Stem Cell Culturing}

A

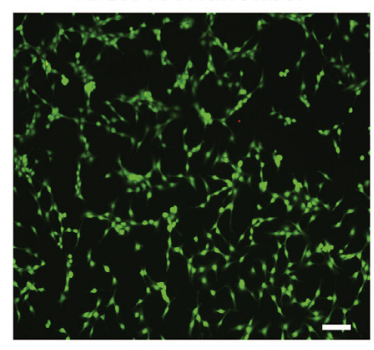

C
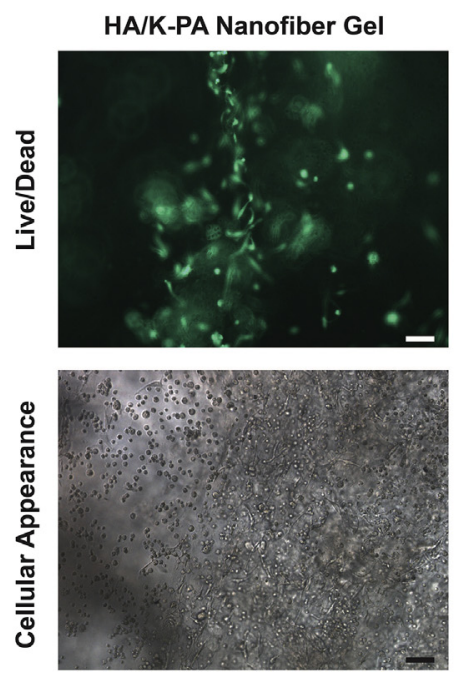

E-PA/K-PA Nanofiber

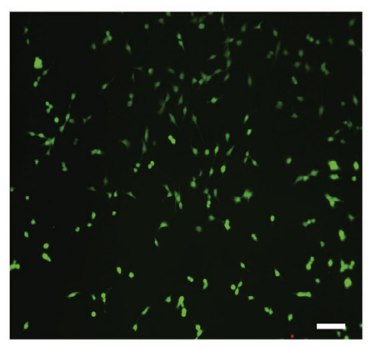

TCP

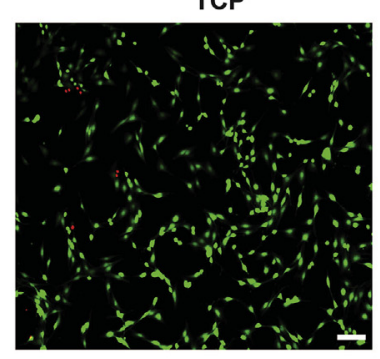

B

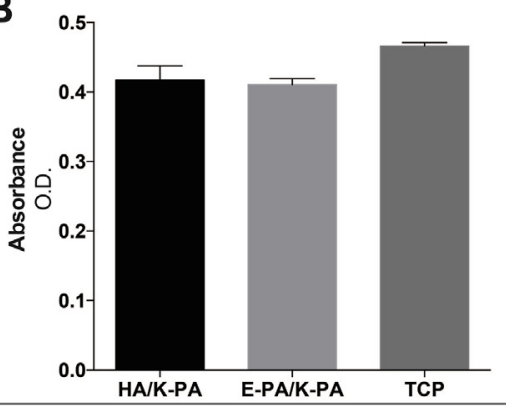

3D In vitro Chondrocyte Culturing in HA/K-PA Nanofiber Gel
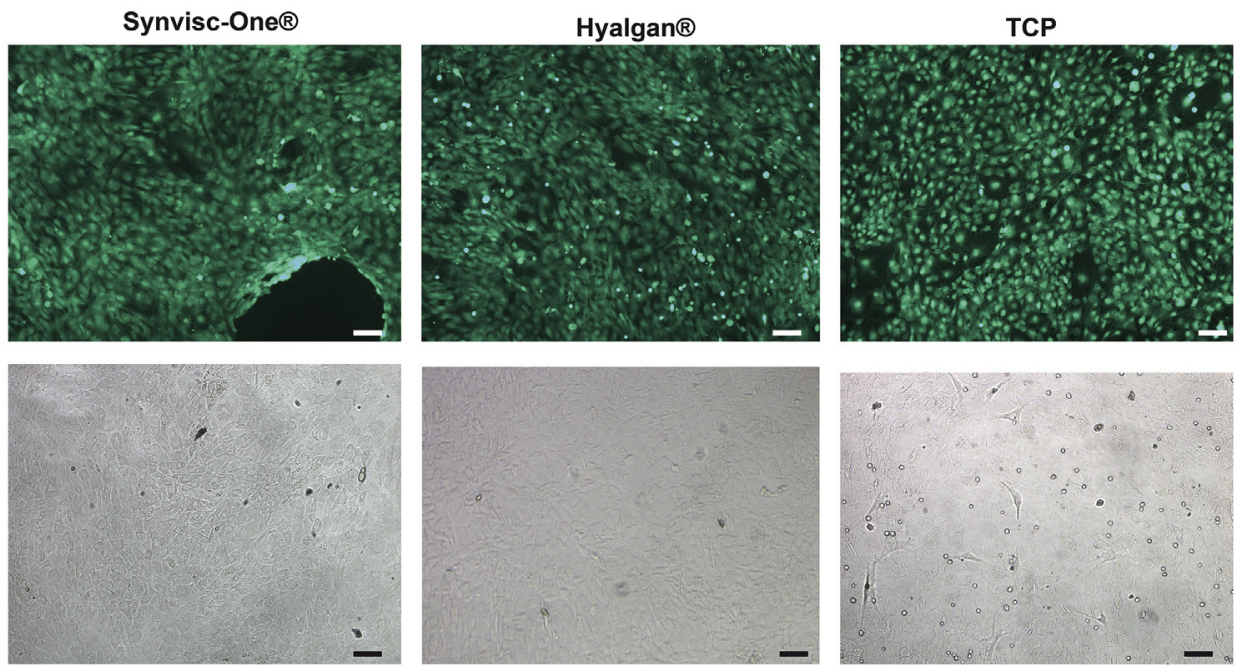

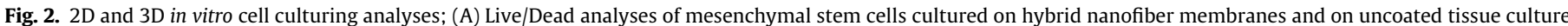

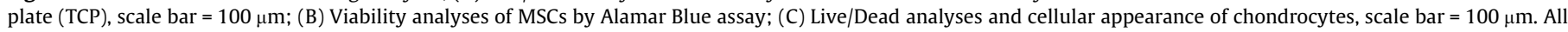
in vitro experiments were performed with at least 5 replicas. 
region of joint after 4-6 weeks [34]. Therefore, 1 week and 6 weeks time windows were chosen as waiting periods after surgery for the two armed-experimental model $(1 \mathrm{~W} / 6 \mathrm{~W}$-early stage OA groups and $6 \mathrm{~W} / 6 \mathrm{~W}$-late stage $\mathrm{OA}$ groups). To evaluate the protection effect on cartilage tissue, all tissues were extracted 6 weeks after injections. Overall, 48 animals were utilized for this study and 46 were included and completed; however, 2 rats in HA/K-PA$1 \mathrm{~W} / 6 \mathrm{~W}$ (early $\mathrm{OA}$ ) group were excluded due to technical difficulties during the surgery.

For the development of OA model, medial meniscus destabilization was performed by the excision of connective tissues between femur and tibial bones in the knees of rats and subsequently $\mathrm{OA}$ formation was observed due to cartilage wear [34]. The medial meniscotibial ligament (MMTL) is the connective tissue that fixes the tibial plateau to the medial meniscus (MM) [34]. MM provides an articular connection between the tibia and femur, as well as helping to transfer body weight on a wider surface, alleviating the load on the cartilaginous body. With the excision of MMTL and destabilization of MM, the MM structure was disturbed and the local mechanical stress increased with the concentration of body weight in a smaller area. In rats, increasing mechanical stress led to injuries at the back of the femur, in the center of the tibia,
A

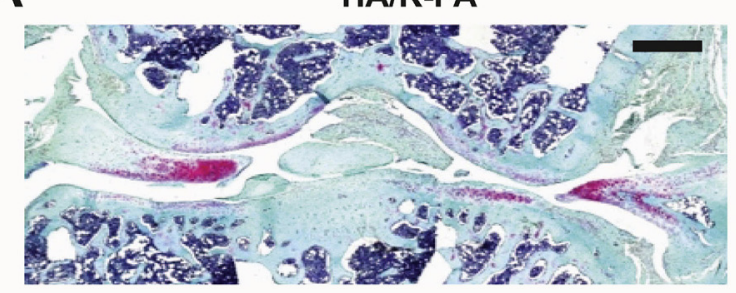

Hyalgan $®$

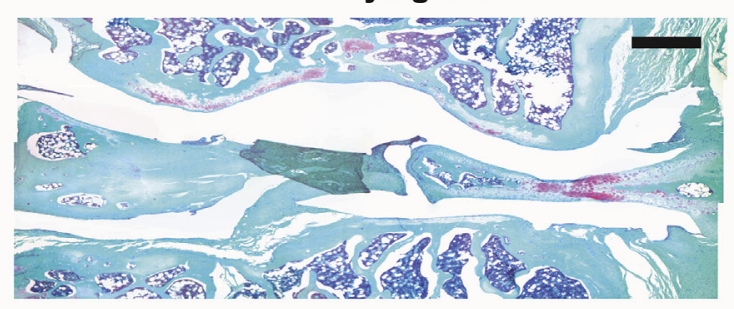

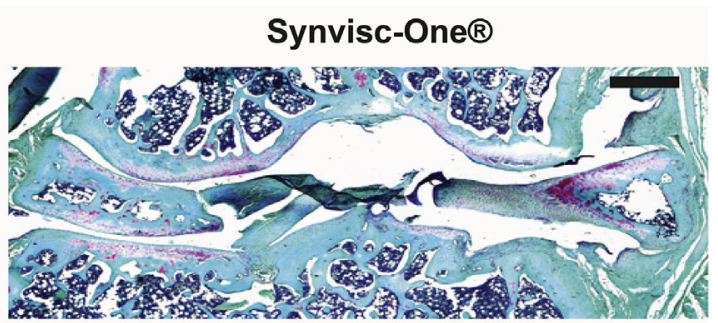

Sham

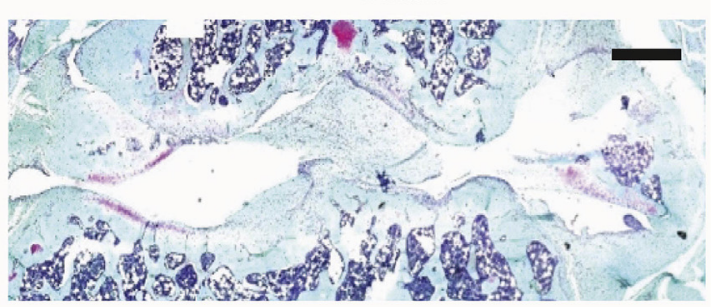

B
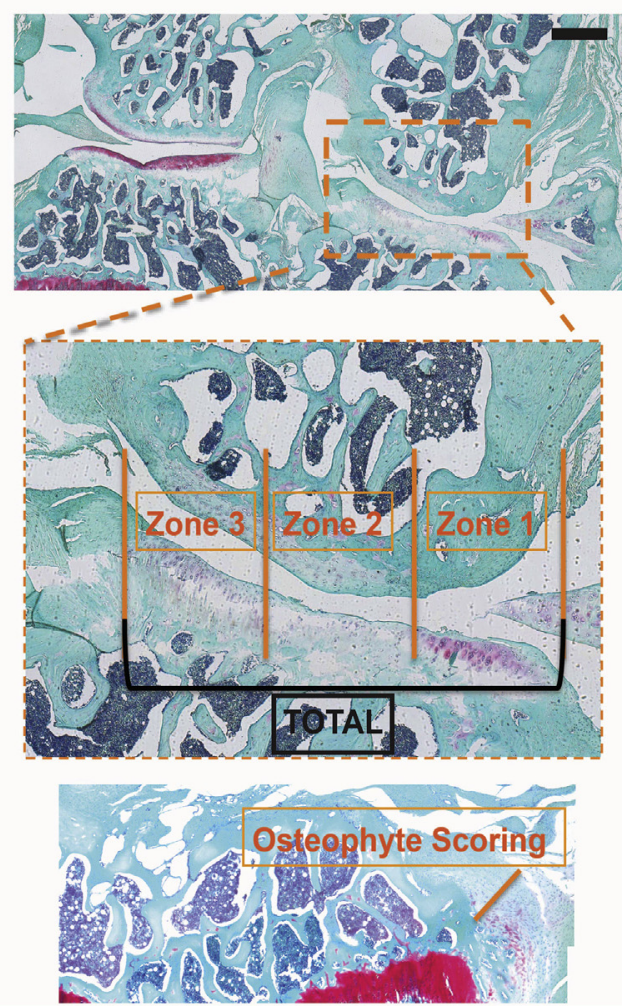

C

\section{Total Joint Degeneration Score}

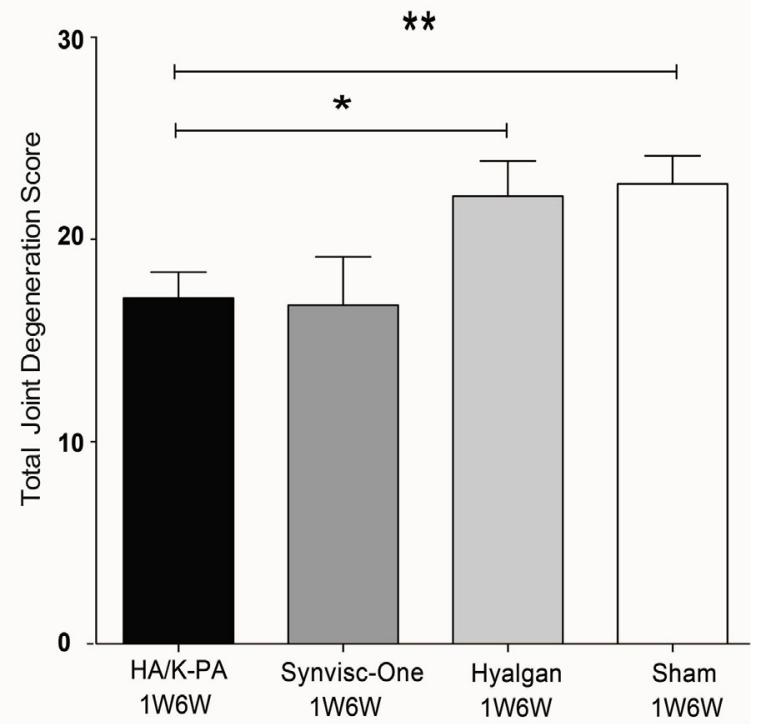

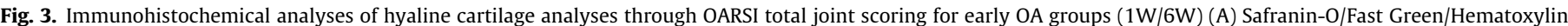

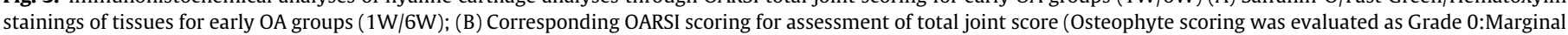

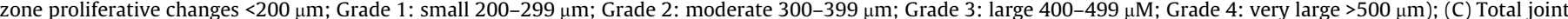

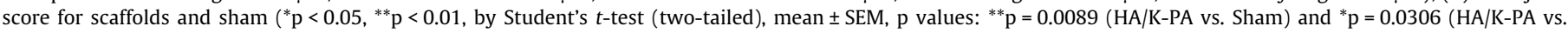
Hyalgan $\left.{ }^{\circledR}\right)$ ). All in vivo experiments were performed with at least 5 replicas. 
and in the medial cartilaginous region. Human osteoarthritis also manifests itself in the medial part of the knee, demonstrating the suitability of this model. Hence, in vivo efficacy of hybrid nanofiber membranes was investigated in osteoarthritic knees of rats.

For the evaluation of osteoarthritic tissue, an OARSI (Osteoarthritis Research Society International)-recommended histopathology scoring method was performed [29]. According to this scoring method, total joint degeneration score was measured including the medial tibial and femur cartilage degeneration scoring, medial tibial osteophyte scoring, and bone scoring. Both early stage and late stage of $\mathrm{OA}$ were modeled using two experimental groups that are $1 \mathrm{~W} / 6 \mathrm{~W}$ (Early group: scaffold injection 1 week after surgery \& tissue extraction 6 weeks after injection) and 6W/6W (Late group: scaffold injection 6 weeks after surgery and tissue extraction 6 weeks after injection) groups. The therapeutic effect of hybrid nanofiber membranes for the protection from degeneration could be assessed through this two-arm experimental method (Fig. S10). For the evaluation of cartilage degeneration scores, tissue sections were stained with Safranin-O/Fast green/ Hematoxylin, and areas of cartilage, where proteoglycan loss was present without any chondrocyte death, were analyzed. Through this scoring, overall cartilage pathology and the important pathological parameters of collagen matrix fibrillation and loss/maintenance of matrix were determined. In addition, the tibial plateau was divided into 3 zones to effectively quantify the degeneration process (Fig. 3). According to total degeneration scoring results, the hybrid nanofiber membrane had the lowest joint degeneration scoring compared to other experimental groups and provided efficient protection to the joint, performing significantly better than commercially available Hyalgan ${ }^{\circledR}$ gel and sham control. In addition, the hybrid nanofiber membrane provided comparable protective effect to Synvisc ${ }^{\circledR}$ sample, which is a crossed-linked, higher molecular weight version of hyaluronic acid that is more expensive and has been shown to exhibit immunogenicity [16,35]. In addition, total degeneration width and significant degeneration width of the medial tibial plateau were evaluated, and the hybrid nanofiber membrane was found to provide enhanced protection from degeneration on cartilage through the surface ( $0 \%$ depth)-midzone (50\% depth)-tide mark (100\% depth) axis (Figs. 4B, S3, S7, S8) than the other scaffolds and sham group. Interestingly, degeneration width measurements demonstrated that the hybrid nanofiber membrane significantly protected cartilage tissue from severe degeneration (Figs. S3F, S7 and S8). Furthermore, zonal depth ratios of lesions were measured and the hybrid nanofiber membrane had the lowest lesion depth, which was significantly lower than Hyalgan ${ }^{\circledR}$ gel and sham control and was comparable with Synvisc ${ }^{\circledR}$ gel (Fig. 4C).

Collagen II immunostaining demonstrated that cells in the hybrid nanofiber membrane-injected tissues had the highest colla-

A

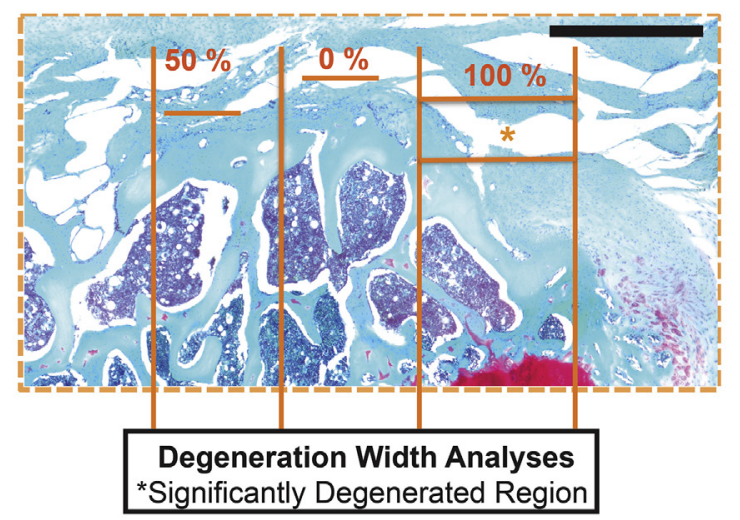

B

C
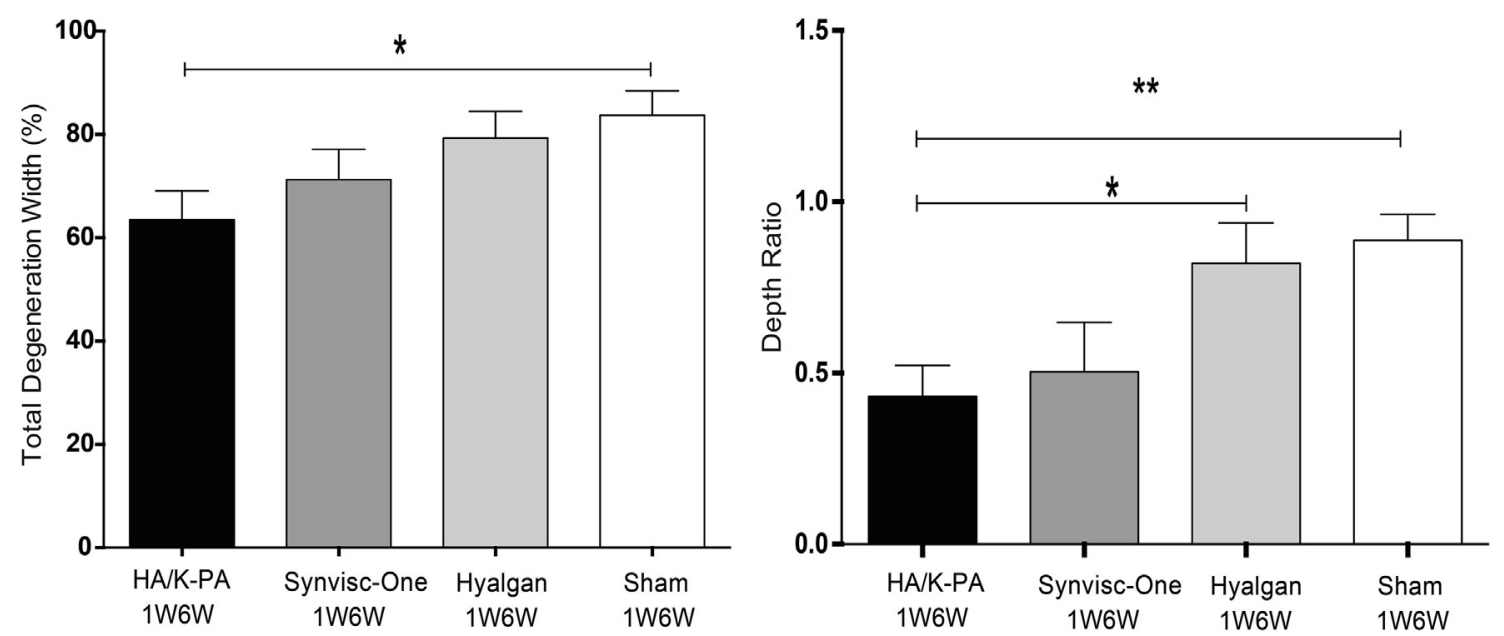

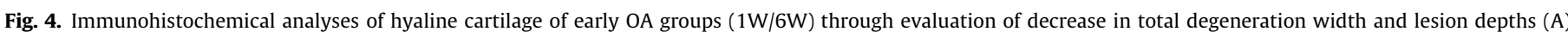

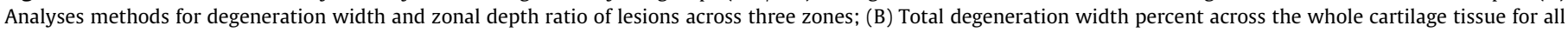

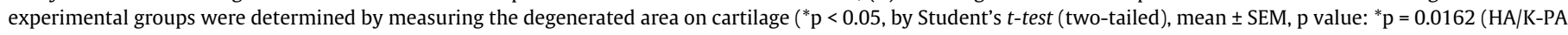

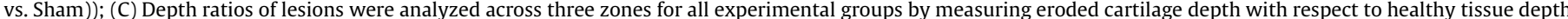

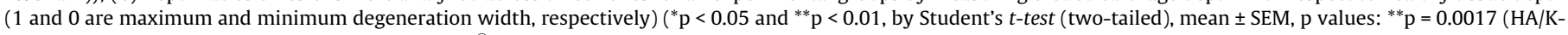
PA vs. Sham) and ${ }^{*} \mathrm{p}=0.0177$ (HA/K-PA vs. Hyalgan $\left.{ }^{\circledR}\right)$ ). All in vivo experiments were performed with at least 5 replicas. 
Collagen II
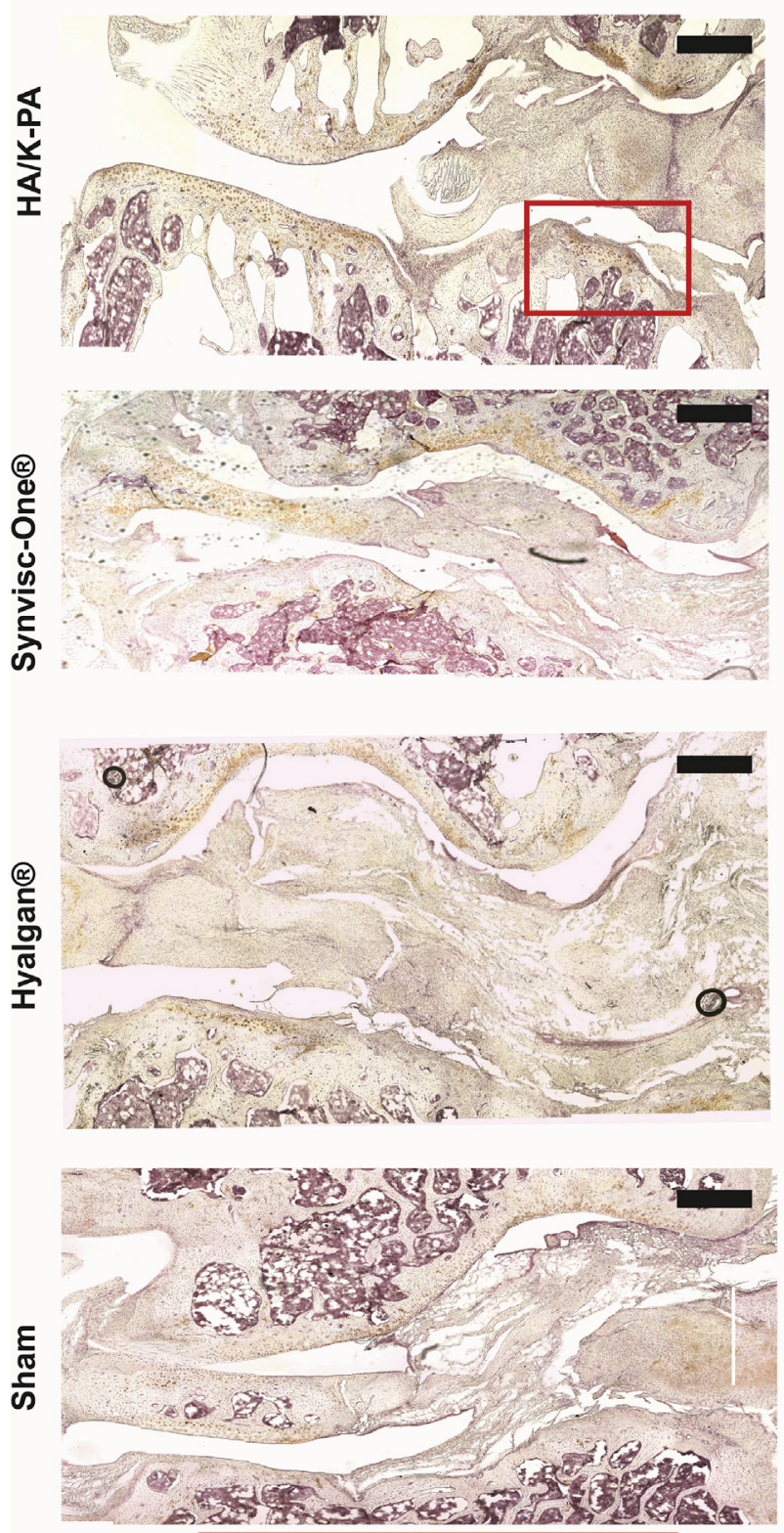

Cartilage Specific Collagen II
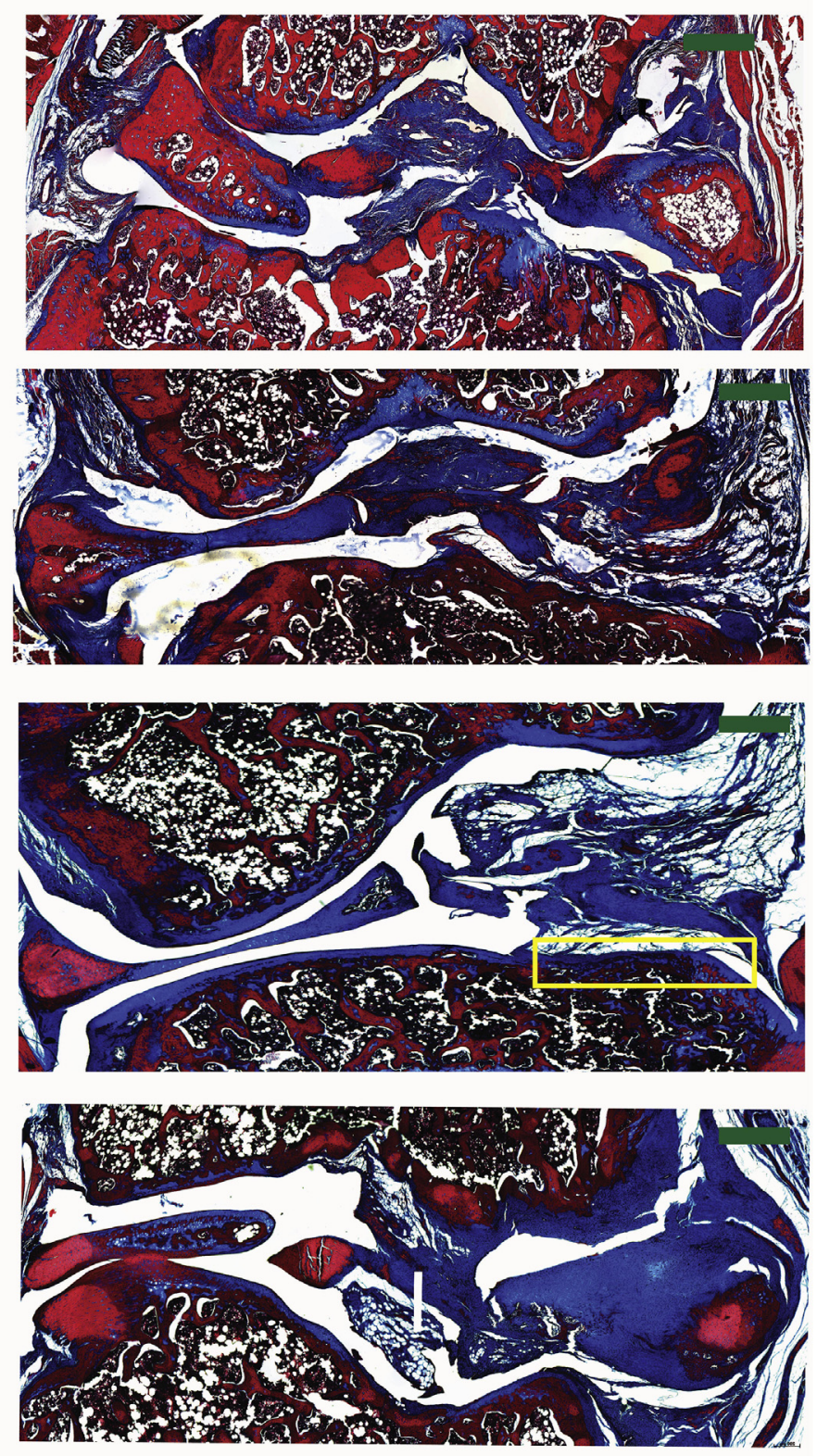

Collagen Fibers Mature Bone \& Muscles

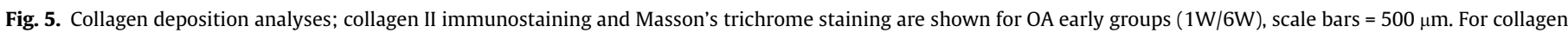

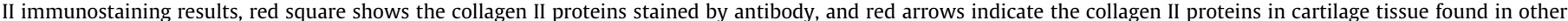

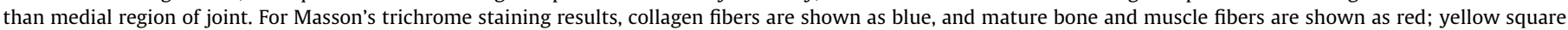
shows the collagen I fiber-rich fibrous cartilage. Magnified images are provided in the supporting file.

gen II deposition, whereas little-to-no collagen II presence was observed in the other groups, especially on the medial part of tibial plateau (Figs. 5 and S9). In addition, Masson's trichrome and Sirius red stainings were also performed to show the collagen structure and deposition on the tissue (Figs. 5 and S5). Masson's trichrome staining results demonstrated the presence and integrity of the different structures of joint capsule, which are shown in the Fig. 5. Moreover, we also showed different structural properties of the joints by using Sirius red staining, which is included in the supporting file (Fig. S5). No signs of inflammation were observed during the main experiments or during optimization studies. In addition, qualitative observations of the sections revealed no abnormality in the synovial membrane structure due to inflammation.

\section{Discussion}

$\mathrm{OA}$ of the knee is an increasingly prevalent condition and has become one of the most common joint diseases in the aged population, as well as in populations having epidemic obesity [18]. Although pain and loss of function associated with OA strongly affect the patients' quality of life, there is no effective treatment against this disease in clinics [18]. Failure of the damaged cartilage matrix to recover is essential for the etiology of OA [18]. In addition, the progressive nature of $\mathrm{OA}$ necessitates early intervention for its effective treatment. When damage occurs at earlier stages of $\mathrm{OA}$, chondrocyte clusters form at the damage site to compensate for matrix degradation through the action of tissue-destructive 
proteinases, and the local concentration of growth factors is increased to enhance the recovery attempt [36-38]. However, cartilage recovery is not effective and ultimately fails, which leads to imbalances in tissue anabolism/catabolism that favor catabolic activity. This process leads to the production of a mechanically weak matrix that cannot withstand the mechanical stresses that cartilage tissue is constantly exposed to. Therefore, especially at the early stages of disease, tissue-engineering strategies could be effective to enhance the recovery attempt of the tissue for the formation of a functional hyaline cartilage matrix. In this study, peptide amphiphile molecules were combined with FDA-approved, clinically used and commercially available Hyalgan ${ }^{\circledR}$, which is composed of high molecular weight hyaluronic acid. Previously, the proposed hybrid nanofiber membrane formation was prepared as mixture of negatively charged another high molecular weight hyaluronic acid with positively charged peptide amphiphile solution [27]. However, in this study, we used Hyalgan ${ }^{\circledR}$ instead in order to better compare its efficiency with a clinically use system. The mixture of Hyalgan ${ }^{\circledR}$ with the positively charged peptide amphiphile solution resulted in formation of hybrid nanofiber membranes with higher hydrolytic stability and mechanical strength even at $\sim 1 / 4$ of the concentration of gels (Hyalgan ${ }^{\circledR}$ and Synvisc (Hylan G-F 20) ${ }^{\circledR}$ ) used in clinics.

$\mathrm{ACI}$ is one of the leading OA treatment options. Considering the essential role of chondrocyte clustering for matrix re-synthesis and recovery at the early stages of OA development, ACI gained considerable attention for enhancing the healing capacity of degenerated matrix [38]. Hence, we also investigated the biocompatibility of the hybrid nanofiber membrane for chondrocyte implantation. The HA/K-PA nanofiber membrane hydrogel was found to support chondrocyte growth and provided a native ECM-like 3D microenvironment. On the other hand, both Hyalgan ${ }^{\circledR}$ and Synvisc ${ }^{\circledR}$ scaffolds, which were the controls for in vivo experiments as well, were hydrolytically unstable and disintegrated into culture media, which prevented the encapsulation of cells in 3D microenvironment. Hyaluronic acid (HA), which is the bioactive molecule presented in these scaffolds, has been previously shown to have biological effects in addition to visco-supplementation, including regulation of cell adhesion, migration, differentiation and proliferation through interaction with CD44, RHAMM, and ICAM-1 receptors [39]. Thus, the hybrid scaffold can not only be used as a physical support system for encapsulation of cells during MACI treatment, but also can provide biological signals for cells similarly to their natural environment [32]. This experiment also shows that just providing biological signals without providing a mechanical support would not be suitable for MACI utilization of these molecules. Since the hybrid nanofiber membrane was shown to provide a suitable microenvironment for stem cell growth, it can also be used in stem cell recruiting approaches for cartilage tissue engineering applications in the future.

The shortcomings of current treatment options necessitate the investigation of possible therapeutics against $\mathrm{OA}$. Hence, the efficiency of HA/K-PA hybrid nanofiber membrane scaffold was analyzed through an in vivo OA model. When these two solutions are combined, a solid membrane is formed which consists of a hierarchically organized nanofibrous structure that is stable, mechanically robust and injectable in addition to presenting bioactive hyaluronic acid molecules. Both early stage and late stage of OA were modeled using two experimental groups: 1W/6W (Early group: scaffold injection 1 week after surgery \& tissue extraction 6 weeks after injection) and 6W/6W (Late group: scaffold injection 6 weeks after surgery \& tissue extraction 6 weeks after injection) groups. According to the immunohistochemical results, the hybrid nanofiber membrane was found to exhibit significantly enhanced protection capacity compared to clinically prescribed gels for early OA groups. When defect was monitored by the staining of repre- sentative slides taken at 20 section $(\sim 200 \mu \mathrm{m})$ intervals to determine the extent of cartilage degeneration through the anterior to posterior regions of cartilage, the cartilage tissue morphology was observed to be preserved better in HA/K-PA injected animals. In addition, the expression of cartilage-specific type II collagen was shown to increase in HA/K-PA group. In addition, collagen localization of cartilage tissue was shown through Mason's trichrome and Sirius red stainings. Although there is collagen deposition in cartilage tissue for Hyalgan ${ }^{\circledR}$ group, this collagen is type I collagen which indicating the fibrous cartilage formation in these animals. This fibrous cartilage showed apparent surface irregularities and compromised the tissue function. The formation of fibrous cartilage was demonstrated through increased collagen I presence based on Masson's trichrome staining results and on the structural irregularities that we observed in all stained tissue sections. Collagen fibrils formed large bundles, which were stained blue by Masson's trichrome staining [40]. The increase in blue staining is attributed to increase in the collagen I presence, which is an important component of fibrous cartilage that we observed especially in Hyalgan $^{\circledR}$ injected tissues. On the other hand, hybrid nanofiber membrane promoted the preservation and formation of hyalinelike cartilage composed of collagen type II and GAGs as opposed to fibrous cartilage. Although we did not find statistically significant differences among late OA groups, we also observed that degeneration score exhibited a downward trend for hybrid nanofiber membrane, which suggests that protection against degeneration was also enhanced for the late OA groups (Fig. S4). The twoarmed experimental model for the in vivo study, which modeled both early and late stages of $\mathrm{OA}$, was used to evaluate the protective effect of the hybrid nanofiber system. We observed enhanced protection potential of the hybrid system in early OA groups. In addition, starting the treatment at early stages of OA was found to be much more efficient as scoring results suggested. The significant decrease in degeneration scoring in HA/K-PA injected tissues was interpreted as protection from degeneration since decreased degeneration scoring was found in both early and late groups. The animal experiments and analyses for early-stage and latestage OA groups were performed separately (Table 1 ). In the initial design of the study, we mainly focused on the early stages of osteoarthritis. After showing that the hybrid gel had significant protective effect from degeneration compared to other gels in the early-OA stage, the late stage of OA was also studied as the second arm of the study (two-armed experimental model) (Fig. S10). Since the experiments for these two groups were designed and performed independently, they were examined separately (comparison of samples was done individually for each group (early or late). In addition, although rodent models for in vivo osteoarthritis study is one of the extensively used cost-effective models, rodent joints have relatively thinner cartilage and open growth plates, which likely increase intrinsic healing potential of the tissue [41]. Thus, we did not compare early and late groups to each other due to independent timing, design, and performance of experiments. We compared the hybrid hydrogel with other gels for each timing group separately, and we mainly focused on the protective therapeutic effect for early stage of OA. The reason of lower degeneration score of samples in the late group could be related to differences among animals and intrinsic healing potential due to longer time past after surgery for late-OA group. In addition, at the early stages of $\mathrm{OA}$, there could be immediate increase in the inflammatory molecules in the cells (such as NF- $\kappa \beta$, TNF $\alpha$, IL $\beta 1$, etc.) as it was also shown in the literature, which could be reason of elevated degeneration scores seen for early OA groups [42]. However, no expression analysis was performed for these molecules. Nevertheless, there were no abnormalities in the synovial membrane structure due to inflammation in any of the samples. According to the staining results, improved cartilage tissue structure maintenance, 
increased chondrocyte presence, and enhanced cartilage specific GAG deposition were interpreted efficient therapeutic effects of HA/K-PA injection. Immunohistochemical stainings showed significantly more collagen II presence in HA/K-PA injected tissues at medial region of the cartilage that was highly affected from OA deterioration, which we interpreted as suppression of loss of collagen II. It was previously shown that suppression of degradation has a substantial role in increasing the cartilage recovery by reducing catabolic reactions [43]. Suppressing chondrocyte death, for example, would preserve the production of cartilage-specific proteins and GAGs, which results in the protection of cartilage structure and functioning [43]. Therefore, delayed degeneration and prolonged protection of cartilage tissue were enhanced by HA/K-PA injection. Moreover, these findings also demonstrated that initiating treatment at early stages of $\mathrm{OA}$ is crucial for the amelioration of disease symptoms.

Hyaluronan is one of the important components of synovial fluid in joints, and provides a viscoelastic environment and sufficient lubrication to reduce wear in cartilage [44]. In addition, it functions as a scaffold by retaining important macromolecules such as aggrecan and link proteins in joints, and stimulates the endogenous synthesis of hyaluronan by interacting with CD44 receptors on chondrocytes [44]. Despite of the fact that Hyalgan ${ }^{\circledR}$ and Synvisc ${ }^{\circledR}$ are prescribed in clinics as hyaluronan derivatives for OA treatment at early/intermediate stages of disease, they have limited and short-term efficiency [17]. On the other hand, the hybrid scaffold both provided bioactivity by stimulating native ECM turnover through the presence of hyaluronic acid, and presented a mechanically stable viscoelastic microenvironment to maintain tissue function.

Self-assembly of molecules at structural interfaces is a major feature for the preparation of chemically well-defined and wellordered materials and presents several opportunities in tissue engineering and clinical applications. It was previously demonstrated that the combination of small synthetic PA molecules with high-molecular weight natural polysaccharide hyaluronic acid results in the formation of a solid membrane at the liquid-liquid interface [27]. Through this mechanism, HA/K-PA scaffold provided a mechanically robust network (Fig. $1 F$ ) due to the hierarchical order of K-PA and HA molecules. A similar membrane system was shown to be more stable than high molecular weight polysaccharide combination chitosan - gellan membranes, which were prone to disintegrating in aqueous environment [27]. In addition, this system allowed the formation of HA-filled membrane sacs that were shown to be robust enough to withstand suturing without tearing [27]. This feature is essential for the efficiency of membranes to be used in cartilage defects, because cartilage is constantly exposed to repetitive mechanical loading. Consequently, tear-resistance is crucial for the proper functioning of tissue substitutes. In addition, this hierarchically self-assembling membrane system facilitated the encapsulation of hyaluronic acid within the sacs, allowing its maintenance for a longer time following injection [27]. However, the use of hybrid PA/hyaluronic acid systems for OA treatment has not been reported to date.

The nanofiber system described in the present study uses the same design principles as previously described hybrid membranes and can be effectively used for long-term treatment of OA. Furthermore, the therapeutic activity of the HA/K-PA membrane was provided at a relatively lower concentration than Hyalgan ${ }^{\circledR}$ and Synvisc ${ }^{\circledR}$ gels, which can be considered for the potential of superior therapeutic effect of this hybrid system at a lower production cost in the future. Hence, improved protection from degeneration of cartilage has been provided by using this unique structure presenting less dosage of hyaluronic acid in hybrid nanofibrous form. Although the hybrid nanofiber membrane scaffold demonstrated comparable protective effect to Synvisc ${ }^{\circledR}$ gel, the latter material is associated with shortcomings due to its cost and immunogenicity problems [35]. In addition, the tailorable features of PAs present a broad opportunity to improve membrane design and enhance therapeutic activity through the incorporation of specific bioactive epitopes. Furthermore, this membrane sac system allows the encapsulation and infiltration of large macromolecules such as proteins and growth factors. Hence, this system can be used for further drug delivery applications such as the delivery of nonsteroidal anti-inflammatory drugs (NSAIDs) against OA.

\section{Conclusion}

Osteoarthritis is associated with severe pain and loss of function and is one of the leading causes of joint disabilities in older adults. The development of effective and novel treatment strategies against this disease is therefore of great importance for restoring damaged cartilage function. In this study, we have examined the in vitro and in vivo efficiency of a hybrid nanofiber membrane and observed that it enabled chondrocyte encapsulation and provided a suitable culturing environment for stem cell growth in vitro, while facilitating the preservation of cartilage morphology with reduced osteophyte formation, protecting the subchondral region from deterioration, and maintaining cartilage-specific matrix proteins in vivo. In addition, this system allows the formation of HA-filled membrane sacs that present a tear-resistant scaffold, which is crucial for the proper functioning of tissue substitutes under repetitive mechanical stress in joints. The hybrid nanofiber membrane system described in this study could therefore protect cartilage tissue from degeneration and enhance the repair process at the site of injury. Overall, the therapeutic activity of the hybrid nanofiber membrane system was provided at a relatively lower concentration than Hyalgan ${ }^{\circledR}$ and Synvisc ${ }^{\circledR}$ gels, which allows its use as a potential candidate for further clinical applications.

\section{Author contributions}

In vivo experiments were designed and performed by E.A., N.C., H.G., H.O., and A.B.T.; in vitro experiments were designed and performed by E.A., M.O.G. and A.B.T.; chemical synthesis and characterizations were designed and performed by E.A., M.S.E., and M.O. G.; stem cell culture experiments were performed by E.A. and C.E.C. The manuscript was written by E.A., M.O.G., and A.B.T., with contributions of all authors. All authors have given approval to the final version of the manuscript.

\section{Acknowledgments}

E. A. acknowledges support from TUBITAK-BIDEB fellowship. We would like to express our gratitude to Mr. M. Guler for assistance in TEM, to veterinarian Mr. M. Ak for assistance in in vivo experiments, and to Dr. A. D. Ozkan and Dr. B. Senturk for valuable comments. A.B.T. acknowledges support from the Science Academy Outstanding Young Scientist Award (BAGEP).

\section{Conflict of interest}

The authors have no competing conflicts of interest to declare.

\section{Appendix A. Supplementary data}

Supplementary data associated with this article can be found, in the online version, at https://doi.org/10.1016/j.actbio.2018.04.015. 


\section{References}

[1] A.J. Sophia Fox, A. Bedi, S.A. Rodeo, The basic science of articular cartilage: structure, composition, and function, Sports Health 1 (6) (2009) 461-468.

[2] D.T. Felson, Y. Zhang, M.T. Hannan, A. Naimark, B. Weissman, P. Aliabadi, D. Levy, Risk factors for incident radiographic knee osteoarthritis in the elderly: the Framingham Study, Arthritis Rheum. 40 (1997).

[3] M. Grotle, K.B. Hagen, B. Natvig, F.A. Dahl, T.K. Kvien, Obesity and osteoarthritis in knee, hip and/or hand: an epidemiological study in the general population with 10 years follow-up, BMC Musculoskeletal Disorders 9 (1471-2474) (2008) 1-5.

[4] J. Clouet, C. Vinatier, C. Merceron, M. Pot-vaucel, Y. Maugars, P. Weiss, G. Grimandi, J. Guicheux, From osteoarthritis treatments to future regenerative therapies for cartilage, Drug Discov. Today 14 (19-20) (2009) 913-925.

[5] D. Prakash, D. Learmonth, Natural progression of osteo-chondral defect in the femoral condyle, Knee 9 (1) (2002) 7-10.

[6] G.E. Gold, S.B. Reeder, H. Yu, P. Kornaat, A.S. Shimakawa, J.W. Johnson, N.J. Pelc, C.F. Beaulieu, J.H. Brittain, Articular cartilage of the knee: rapid threedimensional MR imaging at 3.0 T with IDEAL balanced steady-state free precession-initial experience 1, Radiology 240 (2) (2006) 546-551.

[7] J. Taipale, J. Keski-Oja, Growth factors in the extracellular matrix, FASEB J. 11 (1) (1997) 51-59.

[8] D. Eyre, Collagen of articular cartilage, Arthritis Res. 4 (1) (2002) 30-35.

[9] D. Eyre, Articular cartilage and changes in arthritis: collagen of articular cartilage, Arthritis Res. Therapy 4 (1) (2001) 30.

[10] S.W. Li, M. Takanosu, M. Arita, Y. Bao, Z.X. Ren, A. Maier, D.J. Prockop, R. Mayne, Targeted disruption of Col11a2 produces a mild cartilage phenotype in transgenic mice: comparison with the human disorder otospondylomegaepiphyseal dysplasia (OSMED), Dev. Dyn. 222 (2) (2001) $141-152$.

[11] E. Zamir, B. Geiger, Molecular complexity and dynamics of cell-matrix adhesions, J. Cell Sci. 114 (20) (2001) 3583-3590.

[12] A. Gigout, M. Jolicoeur, M. Nelea, N. Raynal, R. Farndale, M.D. Buschmann, Chondrocyte aggregation in suspension culture is GFOGER-GPP-and $\beta 1$ integrin-dependent, J. Biol. Chem. 283 (46) (2008) 31522-31530.

[13] P.M. van der Kraan, M.-J. Goumans, E.B. Davidson, P. Ten Dijke, Age-dependent alteration of TGF- $\beta$ signalling in osteoarthritis, Cell Tissue Res. 347 (1) (2012) 257-265.

[14] C. Belcher, R. Yaqub, F. Fawthrop, M. Bayliss, M. Doherty, Synovial fluid chondroitin and keratan sulphate epitopes, glycosaminoglycans, and hyaluronan in arthritic and normal knees, Ann. Rheum. Dis. 56 (5) (1997) 299-307.

[15] K.D. Brandt, G.N. Smith, L.S. Simon, Intraarticular injection of hyaluronan as treatment for knee osteoarthritis: what is the evidence?, Arthritis Rheum 43 (6) (2000) 1192-1203.

[16] V. Dasa, M. DeKoven, K. Sun, A. Scott, S. Lim, Clinical and cost outcomes from different hyaluronic acid treatments in patients with knee osteoarthritis: evidence from a US health plan claims database, Drugs Context 5 (2016).

[17] V.M. Goldberg, L. Goldberg, Intra-articular hyaluronans: the treatment of knee pain in osteoarthritis, J. Pain Res. 3 (2010) 51.

[18] J.W.J. Bijlsma, F. Berenbaum, F.P.J.G. Lafeber, Osteoarthritis: an update with relevance for clinical practice, The Lancet 377 (9783) (2011) 2115-2126.

[19] M.R. Baumgaertner, W.D. Cannon, J.M. Vittori, E.S. Schmidt, R.C. Maurer, Arthroscopic debridement of the arthritic knee, Clin. Orthopaedics Related Res. 253 (1990) 197-202.

[20] W.D. Bugbee, F.R. Convery, Osteochondral allograft transplantation, Clin. Sports Med. 18 (1) (1999) 67-75.

[21] T.J. Gill, P.C. McCulloch, S.S. Glasson, T. Blanchet, E.A. Morris, Chondral defect repair after the microfracture procedure a nonhuman primate model, Am. J. Sports Med. 33 (5) (2005) 680-685.
[22] E.B. Hunziker, Articular cartilage repair: basic science and clinical progress. A review of the current status and prospects, Osteoarthritis Cartilage 10 (6) (2002) 432-463.

[23] T. Minas, S. Nehrer, Current concepts in the treatment of articular cartilage defects, Orthopedics 20 (6) (1997) 525-538.

[24] D.W. Hutmacher, Scaffolds in tissue engineering bone and cartilage, Biomaterials 21 (24) (2000) 2529-2543.

[25] E. Arslan, M.O. Guler, A.B. Tekinay, Glycosaminoglycan-mimetic signals direct the osteo/chondrogenic differentiation of mesenchymal stem cells in a threedimensional peptide nanofiber extracellular matrix mimetic environment, Biomacromolecules 17 (4) (2016) 1280-1291.

[26] D. Carvajal, R. Bitton, J.R. Mantei, Y.S. Velichko, S.I. Stupp, K.R. Shull, Physical properties of hierarchically ordered self-assembled planar and spherical membranes, Soft Matter 6 (8) (2010) 1816-1823.

[27] R.M. Capito, H.S. Azevedo, Y.S. Velichko, A. Mata, S.I. Stupp, Self-assembly of large and small molecules into hierarchically ordered sacs and membranes, Science 319 (5871) (2008) 1812-1816.

[28] M. Gosset, F. Berenbaum, S. Thirion, C. Jacques, Primary culture and phenotyping of murine chondrocytes, Nat. Protocols 3 (8) (2008) 1253-1260.

[29] N. Gerwin, A. Bendele, S. Glasson, C. Carlson, The OARSI histopathology initiative-recommendations for histological assessments of osteoarthritis in the rat, Osteoarthritis Cartilage 18 (2010) S24-S34.

[30] C. Phornphutkul, K.-Y. Wu, P.A. Gruppuso, The role of insulin in chondrogenesis, Mol. Cell. Endocrinol. 249 (12) (2006) 107-115.

[31] R.N. Shah, N.A. Shah, M.M.D.R. Lim, C. Hsieh, G. Nuber, S.I. Stupp, Supramolecular design of self-assembling nanofibers for cartilage regeneration, Proc. Natl. Acad. Sci. 107 (8) (2010) 3293-3298.

[32] J.A. Burdick, G.D. Prestwich, Hyaluronic acid hydrogels for biomedical applications, Adv. Mater. 23 (12) (2011).

[33] M. Lutolf, J. Hubbell, Synthetic biomaterials as instructive extracellular microenvironments for morphogenesis in tissue engineering, Nat. Biotechnol. 23 (1) (2005) 47-55.

[34] S. Glasson, T. Blanchet, E. Morris, The surgical destabilization of the medial meniscus (DMM) model of osteoarthritis in the 129/SvEv mouse, Osteoarthritis Cartilage 15 (9) (2007) 1061-1069.

[35] M. Ishikawa, K. Yoshioka, K. Urano, Y. Tanaka, T. Hatanaka, A. Nii, Biocompatibility of cross-linked hyaluronate (Gel-200) for the treatment of knee osteoarthritis, Osteoarthritis Cartilage 22 (11) (2014) 1902-1909.

[36] P.S. Burrage, K.S. Mix, C.E. Brinckerhoff, Matrix metalloproteinases: role in arthritis, Front. Biosci. 11 (1) (2006) 529-543.

[37] M. Aurich, G.R. Squires, A. Reiner, J.A. Mollenhauer, K.E. Kuettner, A.R. Poole, A A. Cole, Differential matrix degradation and turnover in early cartilage lesions of human knee and ankle joints, Arthritis Rheumatol. 52 (1) (2005) 112-119.

[38] M.B. Goldring, The role of the chondrocyte in osteoarthritis, Arthritis Rheumatol. 43 (9) (2000) 1916-1926.

[39] A. Fakhari, C. Berkland, Applications and emerging trends of hyaluronic acid in tissue engineering, as a dermal filler, and in osteoarthritis treatment, Acta Biomater. 9 (7) (2013) 7081-7092.

[40] M.H. Ross, W. Pawlina, Histology, Lippincott Williams \& Wilkins, 2006.

[41] C.R. Chu, M. Szczodry, S. Bruno, Animal models for cartilage regeneration and repair, Tissue Eng. Part B, Rev. 16 (1) (2010) 105-115.

[42] M.J. Benito, D.J. Veale, O. FitzGerald, W.B. van den Berg, B. Bresnihan, Synovial tissue inflammation in early and late osteoarthritis, Ann. Rheum. Dis. 64 (9) (2005) 1263-1267.

[43] W. Zhang, H. Ouyang, C.R. Dass, J. Xu, Current research on pharmacologic and regenerative therapies for osteoarthritis, Bone Res. 4 (2016) 15040.

[44] D.D. Allison, K.J. Grande-Allen, Hyaluronan: a powerful tissue engineering tool, Tissue Eng. 12 (8) (2006) 2131-2140. 